\title{
ASSOCIAÇÃO ENTRE HÁBITOS DE VIDA E RISCO CARDIOVASCULAR EM PESSOAS VIVENDO COM HIV/AIDS
}

\author{
Marcela Antonini ${ }^{1}$, Elizabete Santos Melo², Christefany Régia Braz Costa ${ }^{3}$, Giselle Juliana de Jesus ${ }^{3}$, \\ Elucir Gir ${ }^{4}$, Renata Karina Reis ${ }^{5}$
}

Objetivo: analisar os hábitos de vida e o risco cardiovascular de pessoas vivendo com o vírus da imunodeficiência humana, por meio de fatores sociodemográficos, clínicos e comportamentais. Método: trata-se de estudo transversal, quantitativo, realizado em serviços especializados no atendimento de pessoas vivendo com o vírus da imunodeficiência humana, no período de 2014 a 2016, num município no interior do estado de São Paulo - Brasil. Utilizou-se Escore de Risco de Framingham para estratificação do risco cardiovascular. Resultados: participaram 340 pessoas. Houve associação entre o risco cardiovascular e as variáveis sexo $(p=0,003)$, idade $(p<0,001)$, escolaridade $(p=0,006)$, antecedentes familiares para Hipertensão $(p=0,026)$, Diabetes $(p=0,003)$, Infarto do Miocárdio $(p=0,004)$ e Acidente Vascular Encefálico $(p=0,003)$, o tempo de diagnóstico pelo HIV $(p<0,001)$ e uso de antirretrovirais $(p=0,003)$. Conclusão: os resultados apontaram associações entre o risco cardiovascular e os fatores de risco modificáveis e não modificáveis para ocorrência de doenças cardiovasculares em pessoas vivendo com HIV.

DESCRITORES: Infecções por HIV; Síndrome da imunodeficiência adquirida; Doenças cardiovasculares; Promoção da saúde.

\section{ASSOCIATION BETWEEN LIFE HABITS AND CARDIOVASCULAR RISK IN PEOPLE LIVING WITH HIV/AIDS}

Objective: To analyze the life habits and cardiovascular risk of people living with the human immunodeficiency virus, through sociodemographic, clinical and behavioral factors. Method: Cross-sectional quantitative study carried out in specialized services in the care of people living with the human immunodeficiency virus, from 2014 to 2016, in a municipality in the inland of the state of São Paulo - Brazil. The Framingham Risk Score was used to stratify cardiovascular risks. Results: Three hundred forty (340) individuals participated in the study. There was an association between cardiovascular risk and the variables gender $(p=0.003)$, age $(p<0.001)$, education $(p=0.006)$, family history of hypertension $(p=0.026)$, diabetes $(p$ $=0.003)$, myocardial infarction $(p=0.004)$ and stroke $(p=0,003)$, time elapsed since HIV diagnosis $(p<0.001)$ and time elapsed since the beginning of use of antiretrovirals $(\mathbf{p}=\mathbf{0 . 0 0 3})$. Conclusion: The results revealed associations between cardiovascular risk and modifiable and non-modifiable risk factors for the occurrence of cardiovascular diseases in people living with HIV.

KEYWORDS: HIV infections; Acquired immunodeficiency syndrome; Cardiovascular diseases; Health promotion.

\section{ASOCIACIÓN ENTRE HÁBITOS DE VIDA Y RIESGO CARDIOVASCULAR EN PERSONAS AFECTADAS POR HIV/SIDA}

\begin{abstract}
Objetivo: Analizar hábitos de vida y riesgo cardiovascular en personas afectadas por el virus de inmunodeficiencia humana, aplicando factores sociodemográficos, clínicos y conductuales. Método: Estudio transversal, cuantitativo, realizado en servicios especializados en atención de personas afectadas por el virus de la inmunodeficiencia humana, entre 2014 y 2016, en un municipio del interior del estado de São Paulo - Brasil. Se utilizó Puntuación de Riesgo de Framingham para estratificación del riesgo cardiovascular. Resultados: Participaron 340 personas. Existió asociación entre riesgo cardiovascular y variables: sexo $(p=0,003)$, edad $(p<0,001)$, escolarización $(p=0,006)$, antecedentes familiares de Hipertensión $(p=0,026)$, Diabetes $(p=0,003)$, Infarto de Miocardio $(p=0,004)$ y Accidente Cerebrovascular $(p=0,003)$, tiempo de diagnóstico del $\mathrm{VIH}(p<0,001)$ y uso de antirretrovirales $(p=0,003)$. Conclusión: Los resultados expresaron asociaciones entre el riesgo cardiovascular los y factores de riesgo modificables y no modificables para el surgimiento de enfermedades cardiovasculares en personas afectadas por el VIH.
\end{abstract}

DESCRIPTORES: Infecciones por VIH; Síndrome de Inmunodeficiencia Adquirida; Enfermedades Cardiovasculares; Promoción de la Salud.

${ }^{1}$ Acadêmica do curso de Enfermagem. Escola de Enfermagem de Ribeirão Preto da Universidade de São Paulo. Ribeirão Preto, SP, Brasil.

${ }^{2}$ Enfermeira. Doutoranda do Programa de Pós-Graduação Enfermagem Fundamental da Escola de Enfermagem de Ribeirão Preto da Universidade de São Paulo Ribeirão Preto, SP, Brasil.

${ }^{3}$ Enfermeira. Doutoranda do Programa de Pós-Graduação Enfermagem Fundamental da Escola de Enfermagem de Ribeirão Preto da Universidade de São Paulo. Ribeirão Preto, SP, Brasil.

${ }^{4}$ Enfermeira. Doutora em Enfermagem. Professora Titular da Escola de Enfermagem de Ribeirão Preto da Universidade de São Paulo. Ribeirão Preto, SP, Brasil.

${ }^{5}$ Enfermeira. Doutora em Enfermagem. Professora da Escola de Enfermagem de Ribeirão Preto da Universidade de São Paulo. Ribeirão Preto, SP, Brasil. 


\section{INTRODUÇÃO}

Passados mais de 30 anos do início da epidemia, e apesar de avanços no tratamento e ações preventivas, a infecção pelo vírus da imunodeficiência humana (HIV) ainda persiste no cenário mundial como um sério e desafiante problema de saúde pública. ${ }^{(1)}$

Dentre essas novas propostas, o advento da terapia antirretroviral (TARV) resultou na melhoria da expectativa de vida de Pessoas Vivendo com HIV/aids (PVHA) e, consequentemente, possibilitou melhorias no cotidiano das mesmas. Deste modo, em muitas regiões do mundo, a infecção pelo HIV, que antes era tida como condição aguda, foi redefinida como doença crônica. ${ }^{(2)}$ Assim, comorbidades advindas do próprio tratamento ou como consequência de maior sobrevida têm emergido nesta população, ${ }^{(3)}$ sendo elas mais vulneráveis de acordo com o estilo de vida e comportamentos de risco como tabagismo, alcoolismo, uso de drogas lícitas e até mesmo a obesidade. ${ }^{(4)}$

De acordo com a Joint United Nations Program on HIV/AIDS (UNAIDS- 2016), até dezembro de 2015 havia em média 36,7 milhões de pessoas vivendo com HIV no mundo, sendo que destas 17 milhões chegaram ao final do ano com acesso ao tratamento. ${ }^{(5)}$

No Brasil, o Departamento de Doenças Sexualmente Transmissíveis (DST), AIDS e Hepatites Virais refere que nos últimos cinco anos o país tem registrado uma média de 41,1 mil episódios de AIDS por ano. No período de 1980 a junho de 2016 foram registrados 842.710 casos e apesar da distribuição estar em expansão por todo o território nacional, as regiões Sudeste e Sul mostram-se a frente do restante do país em termos de concentração dos casos, correspondendo, respectivamente, a 53,0\% e 20,1\% do total identificado. $^{(6)}$

Concomitantemente, a literatura refere que a infecção pelo HIV e o uso de TARV desencadeiam respostas inflamatórias e alterações metabólicas, tais como diabetes por resistência insulínica, dislipidemia devido à alteração de lipoproteínas circulantes, lipodistrofia e produção de quimiocinas, que refletem alterações no endotélio vascular que contribuem para ocorrência de processos ateroscleróticos e, consequentemente, eventos cardiovasculares indesejáveis como hipertensão e doenças coronarianas. ${ }^{(4,7-9)}$

Além disso, as últimas décadas têm representado um período de mudanças nas condições de vida e de saúde da população, a qual está em acentuado processo de envelhecimento e com profundas transformações na qualidade e quantidade dos alimentos ingerido, levando à consequência de maior exposição aos fatores de risco relacionados às doenças crônicas não transmissíveis, principalmente as Doenças Cardiovasculares (DCV). ${ }^{(10)}$

A Organização Mundial de Saúde (OMS - 2003) classifica os fatores de risco que se associam à etiologia das doenças não-transmissíveis em dois grupos, um vinculado ao indivíduo e outro à comunidade. Os fatores de risco do indivíduo estão agrupados como geral, associados ao estilo de vida, e intermediários ou biológicos. No primeiro agrupamento encontram-se idade, sexo, escolaridade e herança genética. No segundo, tabagismo, dieta inadequada e sedentarismo. No terceiro, hipertensão arterial, obesidade e hipercolesterolemia. Entre os fatores de risco associados à comunidade estão as condições socioeconômicas, culturais, ambientais e de urbanização. ${ }^{(11)}$

As condições socioeconômicas são relevantes para os fatores de risco modificáveis, uma vez que influenciam comportamentos e acesso a serviços e bens, os quais podem contribuir ao aparecimento de complicações cardiovasculares. ${ }^{(12-13)}$

No Brasil, a Sociedade Brasileira de Cardiologia trouxe em 2013 que a realidade epidemiológica no país apresenta índices alarmantes de 30\% de mortes por DCV dentre todas as outras causas relacionadas. Apesar das doenças de caráter cardiovascular não serem de notificação compulsória, seus índices alarmantes indicam a necessidade de abordar hábitos de vida, tais como alimentação, tabagismo, etilismo e sedentarismo, com a finalidade de prevenir e orientar a população a respeito de sua condição de protagonista de saúde. ${ }^{(14)}$

Com relação às DCV em PVHA, o paradigma tradicional para explicar o risco aumentado inclui o próprio efeito da infecção pelo HIV, pois apresenta persistente ativação imunológica e inflamatória, associado aos efeitos da TARV em causar dislipidemia, acúmulo ectópico de gordura, diabetes 
ou resistência insulínica. Destacam-se, também, fatores como tabagismo, coinfecções e uso de drogas. ${ }^{(8)}$ Desta forma, tais efeitos e fatores de risco contribuem para a formação de placas coronarianas calcificadas, resultando em eventos coronários. ${ }^{(3)}$

Diante do exposto, nota-se a importância em expandir e aprimorar o conhecimento a respeito dos fatores de risco cardiovasculares modificáveis em PVHA em uso de TARV, para que profissionais da saúde possam aconselhar e elaborar estratégias mais efetivas para o cuidado e melhorar a qualidade de vida desta população, por meio de uma ótica assistencial não só focada na resolução de problemas, mas voltado para a promoção e prevenção em saúde de seus clientes.

Assim, tivemos como objetivo analisar os hábitos de vida e o risco cardiovascular de pessoas vivendo com HIV/aids através dos fatores sociodemográficos, clínicos e comportamentais desta população.

\section{METODOLOGIA}

Trata-se de um estudo transversal, com abordagem quantitativa, realizado em Serviços de Atendimento Especializados no atendimento a PVHA, em um município no interior do estado de São Paulo - Brasil, no período de 2014 a 2016.

Para seleção da amostra, foram convidados a participarem do estudo pessoas vivendo com HIV/aids, de ambos os sexos, que estivessem dentro dos seguintes critérios de inclusão: conhecer sua condição sorológica, independente do estágio de infecção; ter idade superior a 18 anos; estar em uso da TARV instituída a mais de seis meses; estar em acompanhamento clínico-ambulatorial no serviço escolhido e comparecer aos retornos médicos agendados no período de estudo.

Constituíram como critérios de exclusão: os indivíduos em situações de confinamento, tais como presidiários e institucionalizados, residentes em casas de apoio, gestantes, e os indivíduos que já tinham apresentado alguma manifestação de doença cardiovascular.

Os dados foram coletados por meio de entrevistas individuais, em salas do próprio ambulatório, antes ou após a consulta médica e/ou de enfermagem. Após a entrevista, foi realizada mensuração dos dados antropométricos (peso, altura), verificação da pressão arterial, cálculo de IMC e, posteriormente, consulta dos prontuários para obtenção de dados clínicos e laboratoriais, tais como contagem de células TCD4, carga viral, tempo de diagnóstico pela infecção e de tratamento, e demais exames bioquímicos.

Para avaliação das variáveis de interesse, foram utilizados os instrumentos de avaliação do risco cardiovascular, de caracterização sociodemográfica, clínico e comportamental, e avaliação da alimentação saudável, construído pelo Ministério da Saúde, por meio da Política Nacional de Alimentação e Nutrição. O instrumento de caracterização sociodemográfica, clínico e comportamental foi validado por quatro especialistas na área quanto a sua forma e conteúdo, para avaliar a adequação do instrumento quanto aos objetivos do estudo. Após a avaliação, o instrumento foi modificado de acordo com as sugestões dos experts.

O teste "Como está sua alimentação?" avalia as porções do grupo das frutas, hortaliças e cereais, carne, leguminosas e leite, alimentos ricos em gorduras e açúcares; tipo de gordura utilizada para cozinhar; consumo de gordura saturada e colesterol, e sal de adição; número de refeições; ingestão de água e de bebidas alcoólicas; o uso de informações nutricionais dos rótulos de alimentos como orientação para alimentação saudável; e prática de atividade física.

É um instrumento autoaplicado com 18 perguntas que em seu conjunto avaliam a qualidade da dieta. A pontuação total é obtida pela soma de todas as questões, sendo cada uma com um peso diferente (valor mínimo possível 1, valor máximo possível 58). O resultado pode ser menor que 28 pontos, o que significa um escore baixo para alimentação saudável; entre 29 e 42 pontos é um escore intermediário para alimentação saudável; acima de 43 pontos é um escore satisfatório para alimentação saudável.

Para análise do risco cardiovascular, foi utilizado o Escore de Risco de Framingham (ERF). A pontuação total leva em consideração as seguintes variáveis: sexo, idade, tabagismo, diabete mellitus, colesterol total, pressão arterial sistólica e pressão arterial diastólica. O escore obtido corresponde a um 
percentual de probabilidade de ocorrência de doença cardiovascular nos próximos dez anos e são classificados nas categorias: baixo risco - que apresenta uma probabilidade menor que $10 \%$ de eventos cardiovasculares em dez anos; médio risco, entre 10 e 20\%; e alto risco, mais de $20 \%$.

Para a análise dos dados, foi construído um banco de dados em planilha do Excel for Windows, e após a validação da planilha corrigida, os dados foram transportados para o banco de dados definitivo, utilizando o programa de software Statistical Package for Social Science (SPSS), versão 17.0.

Foi realizada estatística descritiva e os testes Qui-quadrado e Exato de Fisher para análise de associação das variáveis relacionadas aos hábitos de vida com o risco cardiovascular. Foi adotado como nível de significância o valor de $p<0,05$.

O projeto foi aprovado pela Secretaria Municipal de Saúde de Ribeirão Preto e pelo Comitê de Ética em Pesquisa da Escola de Enfermagem de Ribeirão Preto - Universidade de São Paulo, conforme protocolo CAAE n.o 34497414.0.0000.5393.

A todos os participantes foi garantido o caráter sigiloso das informações e o anonimato. A coleta de dados foi realizada somente após a concordância dos sujeitos, em uma sala que resguardasse a privacidade dos mesmos, utilizando o Termo de Consentimento Livre e Esclarecido, seguindo os preceitos preconizados pelo Conselho Nacional de Saúde, através da Resolução 466/2012.

\section{RESULTADOS}

Participaram do estudo 340 pessoas vivendo com HIV/aids, sendo 193 (57,9\%) do sexo masculino, 158 (46,5\%) brancos e 228 (67\%) possuíam idade superior a 39 anos. Do total, 280 (82,4\%) vivem com renda mensal inferior a três salários mínimos, $147(43,2 \%)$ eram solteiros e $239(70,3 \%)$ relataram prática heterossexual, conforme os dados apresentados na Tabela 1.

Tabela 1 - Distribuição das pessoas vivendo com HIV/aids, segundo variáveis sociodemográficas, Ribeirão Preto, SP, Brasil, 2014 - 2016. (continua)

\begin{tabular}{llcc}
\hline Variáveis & Categoria & $\mathbf{n}$ & \% \\
\hline \multirow{2}{*}{ Sexo } & Masculino & 197 & 57,9 \\
& Feminino & 143 & 42,1 \\
\hline \multirow{3}{*}{ Idade (em anos) } & $<39$ & 112 & 32,9 \\
& $40-59$ & 197 & 57,9 \\
& $>60$ & 31 & 9,1 \\
\hline \multirow{3}{*}{ Cor } & Branco & 158 & 46,5 \\
& Preto & 46 & 13,5 \\
& Amarelo & 14 & 4,1 \\
\multirow{2}{*}{ Escolaridade (anos de estudo) } & Pardo & 121 & 35,6 \\
& Indígena & 01 & 0,3 \\
\hline \multirow{2}{*}{ Estado Civil } & Menos 8 anos & 167 & 49,1 \\
& Mais 8 anos & 173 & 50,9 \\
\hline \multirow{2}{*}{ Renda (salário mínimo) } & Solteiro & 147 & 43,2 \\
& Casado & 120 & 35,3 \\
& Separado & 39 & 11,5 \\
& Viúvo & 34 & 10 \\
\hline
\end{tabular}




\begin{tabular}{|c|c|c|c|}
\hline \multirow{2}{*}{ Ocupação } & Ativo & 193 & 56,8 \\
\hline & Inativo & 147 & 43,2 \\
\hline \multirow{3}{*}{ Orientação sexual } & Heterossexual & 239 & 70,3 \\
\hline & Homossexual & 82 & 24,1 \\
\hline & Bissexual & 19 & 5,6 \\
\hline Total & & 340 & 100 \\
\hline
\end{tabular}

Quanto as variáveis sociodemográficas e o risco cardiovascular houve associação entre sexo ( $p=0,003)$, idade $(p=0,000)$, escolaridade $(p=0,006)$ e situação de trabalho $(p=0,007)$, conforme apresentado na Tabela 2.

Tabela 2 - Associação entre as variáveis demográficas, hábitos de vida e o Escore de Framingham de PVHA no município de Ribeirão Preto, SP, Brasil, 2014 - 2016

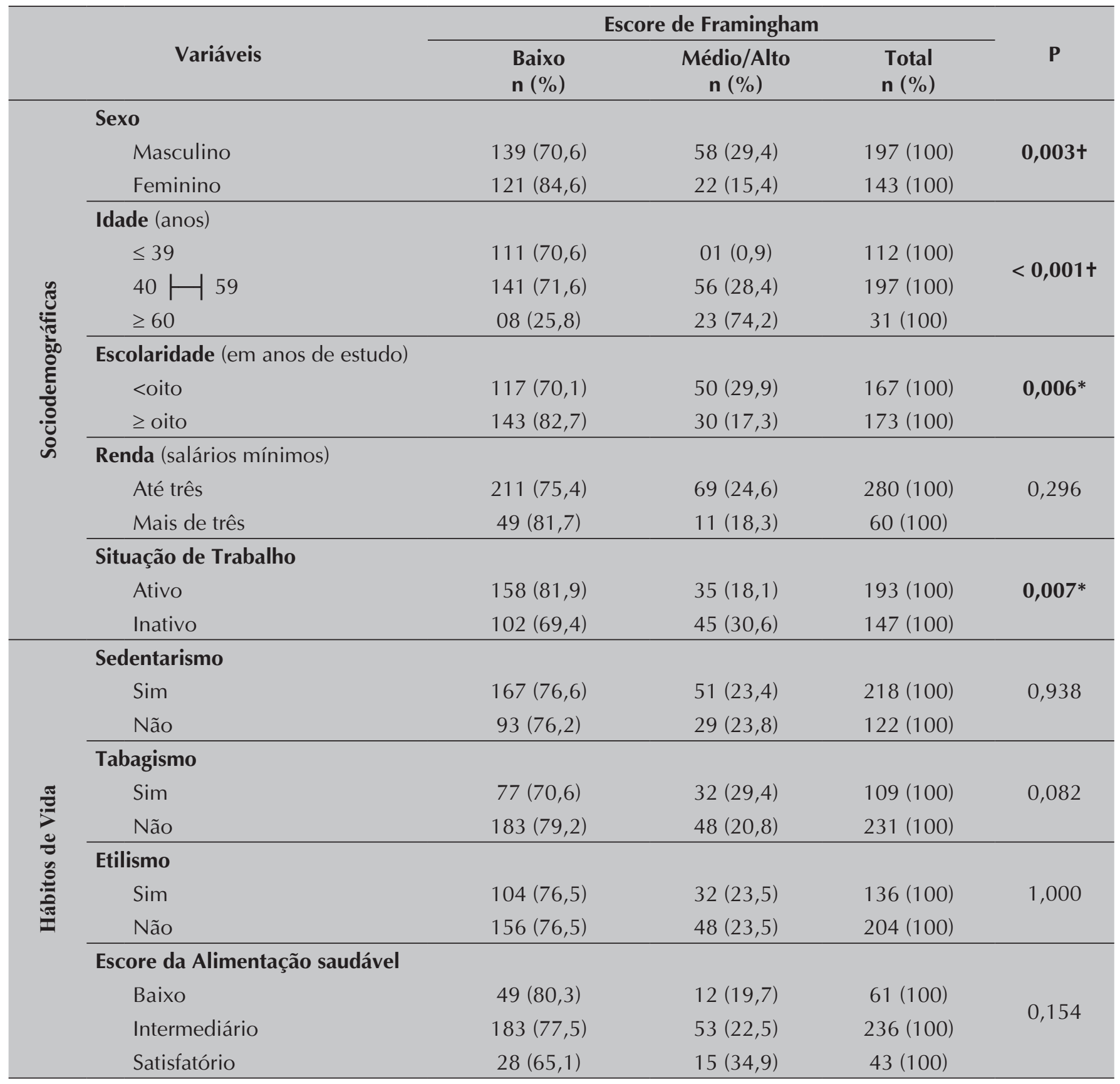

* Teste Qui-Quadrado + Teste Exato de Fisher 
Com relação ao risco cardiovascular e os antecedentes familiares houve destaque nas variáveis de antecedentes familiares para Hipertensão Arterial Sistêmica (HAS) $(p=0,026)$, Diabetes Mellitus (DM) $(p=0,003)$, Infarto Agudo do Miocárdio (IAM) $(p=0,004)$ e Acidente Vascular Encefálico (AVE) ( $p=0,003)$.

Do mesmo modo, dentre as variáveis clínicas relacionadas ao HIV, houve associação significativa com o tempo de diagnóstico $(p=0,000)$ e com o tempo de TARV $(p=0,003)$, conforme indicado na Tabela 3.

Tabela 3 - Associação entre as variáveis clínicas gerais e relacionadas ao HIV e o Escore de Framingham de PVHA no município de Ribeirão Preto, SP, Brasil, 2014 - 2016

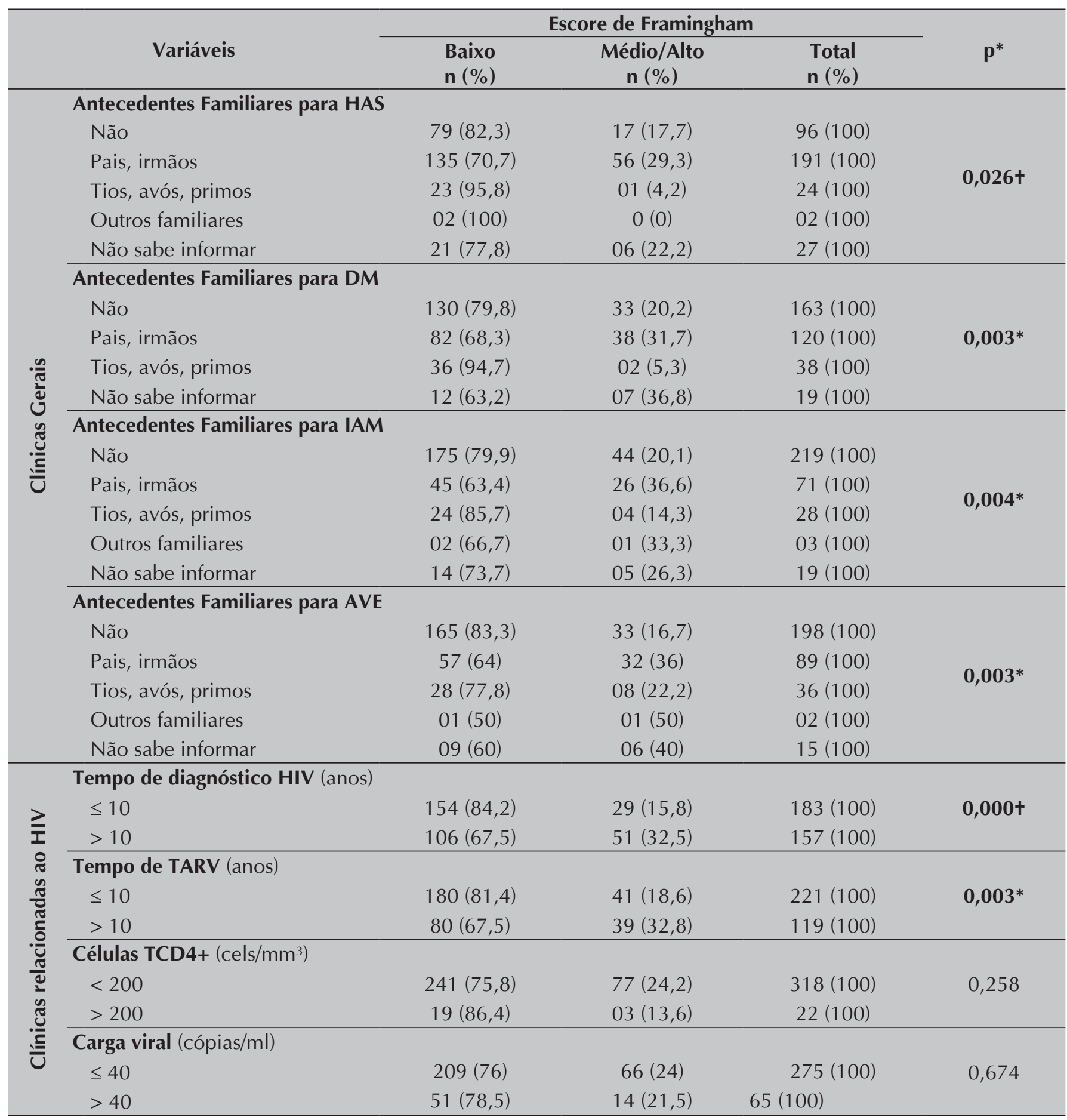

* Teste Qui-Quadrado + Teste Exato de Fisher 


\section{DISCUSSÃO}

Com o passar dos anos o perfil da epidemia tem se diversificado, acarretando novos desafios no que diz respeito às ações preventivas relacionadas ao HIV/aids. ${ }^{(1)}$ No presente estudo, a amostra foi predominantemente do sexo masculino, na faixa etária superior a 39 anos, solteiros e com prática heterossexual. Estes dados estão em harmonia com os resultados apresentados em outro estudo realizado no município de Ribeirão Preto, no qual as características sociodemográficas da amostra são similares. ${ }^{(15)}$ Assim, é possível notar que a epidemia do HIV depara-se com um quadro marcado pelos processos de heterossexualização, interiorização e a pauperização, no qual se destaca o crescimento da epidemia em cidades menores e em populações com menor poder aquisitivo, respectivamente.

Além disso, observou-se que 56,8\% dos participantes estavam ativos no mercado de trabalho, porém, do total, $82,4 \%$ relataram viver com renda mensal abaixo de três salários mínimos e $49,1 \%$ possuíam menos de oito anos de estudo. Do mesmo modo, houve resultado significativo na associação das variáveis escolaridade $(p=0,006)$ e ocupação $(p=0,007)$ com o ERF. Desta forma, torna-se imprescindível discutir fatores de risco modificáveis para DCV, levando em consideração as condições socioeconômicas de um indivíduo.

A pauperização da epidemia abriu espaço para a discussão a respeito da associação da renda familiar com o adoecimento cardiovascular, uma vez que fatores relacionados à pobreza contribuem para comportamentos adversos que influenciam nos fatores de risco, já que a desigualdade econômica é favorável à piora da qualidade de vida, posto que limita a oportunidade de acesso a serviços e bens, contribuindo para o aparecimento de complicações cardiovasculares. ${ }^{(12-13)}$

Assim, é necessário refletir em quais condições econômicas vive esta clientela e qual o acesso desta aos recursos e bens que promovem o bem-estar da saúde, sendo assim imprescindível a familiarização da relevância cultural e socioeconômica de cada individuo pela equipe de saúde, a fim de elaborar assistência mais efetiva e integral e também sob a óptica de prevenção de saúde, mas não apenas focada no diagnóstico e tratamento da infecção.

Destaca-se, ainda, que os indivíduos mais velhos ( $\geq 60$ anos de idade) tiveram maiores escores de risco que os mais jovens ( $p=0,003)$, sendo este dado importante, uma vez que trata-se de uma variável não modificável. Chama atenção que $57,9 \%$ são do sexo masculino, e, portanto, faz-se importante considerar que há diferença na procura pelos serviços de saúde entre homens e mulheres, sendo que os primeiros apresentam atraso na procura do cuidado na atenção básica ou com finalidade preventiva, resultando na procura da atenção especializada, devido à piora ou complicações de doenças. ${ }^{(15)}$

Tal fato nos remete à reflexão de como elaborar estratégias educativas e preventivas com amplitude suficiente para atingir indivíduos distanciados do serviço de saúde e aproveitar o acompanhamento clínico-ambulatorial desta população para intervenções preventivas. Portanto, dirigir intervenções educativas voltadas para esta população mostra-se particularmente importante.

Na Tabela 2 é possível visualizar a associação $(p<0,001)$ da idade com o ERF. Tal dado também foi visto em outro estudo, o qual evidenciou aumento na incidência de Infarto Agudo do Miocárdio (IAM) em indivíduos com HIV/aids, recebendo TARV, de 0,3\% para 1,07\%. ${ }^{(16)}$ Nesse mesmo estudo, identificou-se, ainda, que indivíduos com idade entre 60 e 75 anos apresentaram elevação do risco cardiovascular mais significativa, quando comparados com os mais jovens. ${ }^{(16)}$

Sabe-se que o avançar da idade, por si só, aumenta o risco para doenças crônicas não transmissíveis, dentre elas as cardiovasculares, tornando claro a importância de atuar sobre os fatores modificáveis, de modo a reduzir os riscos e melhorar a qualidade de vida desta população. ${ }^{(17)}$

Além disso, neste estudo foi identificada associação entre os antecedentes familiares para HAS $(p=0,026), \operatorname{DM}(p=0,003), \operatorname{IAM}(p=0,004)$ e AVE $(p=0,003)$ para com o risco cardiovascular, confirmando a evidência da relação entre hereditariedade e as DCV. Tais dados corroboram com um estudo realizado na China, o qual evidenciou que no grupo de PVHA as variáveis idade avançada, excesso de peso e história familiar de doenças cardiovasculares também foram associados ao maior risco cardiovascular. 
(18) Portanto, faz-se necessário a análise dos parentes de primeiro grau de qualquer paciente que venha manifestar DAC numa idade precoce.

Em relação às variáveis clínicas específicas de PVHA, a avaliação dos fatores de risco de DCV nessa população necessita da inclusão da própria infecção pelo HIV junto das demais variáveis. ${ }^{(8)}$ A associação do tempo de diagnóstico do HIV com o Escore de Framingham $(p<0,001)$ condiz com dados da literatura que relacionam o tempo de infecção pelo HIV como um fator de risco para DCV, uma vez que já se sabe que a infecção, por si só, desencadeia a constante ativação imune e persistente resposta inflamatória no endotélio vascular, ativado pela produção de quimiocinas, moléculas de adesão, monócitos e linfócitos $T$, que iniciam processos que contribuem crucialmente para um processo aterogênico e/ou alterações metabólicas como diabetes tipo 2 e dislipidemia. ${ }^{(7-9)}$

Neste mesmo contexto, citocinas como a Interleucina-6 (IL-6), Fator de Necrose Tumoral Alfa (TNF- $\square$ ) e proteína C-reativa (PCR) têm sido referidas como sendo superiores em PVHA e naqueles com imunossupressão grave, e estão relacionadas com o desenvolvimento da aterosclerose, DCV, imunossenescência e mortalidade, devido à não-comorbidades associadas ao HIV.(4)

A associação entre o tempo de TARV com o risco de DCV também apresentou associação significativa $(p=0,003)$. Sabe-se que a TARV aumentou a expectativa de vida de PVHA, porém, junto dela começam a aparecer os efeitos no organismo da combinação tratamento/vírus. Dentre os efeitos da TARV, as alterações no metabolismo dos lipídios podem acarretar alterações metabólicas como a hipertrigliceridemia e a hipercolesterolemia que são fatores de risco para doença cardiovascular. ${ }^{(19)}$ Além disso, a disfunção endotelial é mais uma consequência das alterações metabólicas que podem trazer efeitos deletérios para o coração. ${ }^{(9)}$

No Brasil, o Ministério da Saúde recomenda a realização do ERF uma vez ao ano em PVHA. ${ }^{(20)}$ No presente estudo, aproximadamente $23 \%$ dos participantes apresentaram risco médio/alto segundo o ERF, o que se aproxima dos achados de outro estudo, no qual um terço da amostra teve mais de $10 \%$ de risco de ter uma DCV nos próximos 10 anos. ${ }^{(3)}$ A partir disto, entende-se que esta é uma população com risco potencial para as DCV.

Assim, o ERF é uma ferramenta de baixo custo, rápida e pode ser utilizada durante a consulta de maneira simples e eficaz, tornando-se relevante a sua implementação na assistência de um cuidado sistematizado e integral, ${ }^{(21)} \mathrm{com}$ a finalidade de reduzir, prevenir e detectar precocemente a ocorrência de DCV.

Desta forma, todas as PVHA devem ser rastreadas quanto a fatores de risco cardiovascular, tais como hipertensão e dislipidemia, e devem receber aconselhamento sobre hábitos alimentares saudáveis, exercício físico e cessação do tabagismo. ${ }^{(22)}$

Para tanto, é preciso a sensibilização e capacitação dos profissionais de saúde que atuam na prática clínica, visto que apesar dos Serviços de Atendimento Especializado (SAE) para pessoas que vivem com HIV disporem de equipe multiprofissional, não são encontrados relatos de que a atuação ocorra de forma interdisciplinar. ${ }^{(23)}$

Assim, faz-se necessário discutir sobre estratégias mais efetivas para o cuidado com o objetivo de melhorar a qualidade de vida desta população, através de uma ótica assistencial não só focada na resolução de problemas, mas voltado para a promoção e prevenção em saúde de seus clientes, deixando como desafio a educação continuada e a sensibilização dos profissionais de saúde para a busca deste novo paradigma da infecção.

Além disso, embora variáveis como sedentarismo, tabagismo, etilismo, alimentação saudável, contagem de células TCD4 e carga viral não tenham apresentado associação significativa para as DCV, segundo o ERF neste estudo, destacamos a necessidade de incluí-las na avaliação integral e rotineira de pessoas que vivem com HIV/aids, de modo a trabalhar com medidas preventivas, pois as mesmas são consideradas fatores de risco clássicos para o desenvolvimento de doenças cardiovasculares. 


\section{CONCLUSÃO}

A avaliação dos fatores sociodemográficos, clínicos, comportamentais e do risco cardiovascular identificou que o sexo, idade, escolaridade, situação de trabalho, antecedentes familiares para HAS, DM, IAM, AVE, tempo de diagnóstico do HIV e tempo de uso de TARV apresentaram associação significativa para as DCV, segundo o ERF.

Diante do exposto, este trabalho contribui para o desenvolvimento de um novo olhar na assistência integral à pessoa que vive com HIV/aids. Além disso, fica evidente que as PVHA necessitam de intervenções que abordem seus hábitos de vida como fatores de risco para o desenvolvimento de DCV. É necessário incentivar mudanças nos hábitos alimentares, promoção de atividade física, encorajar a cessação do fumo e do etilismo e dar assistência a sua manutenção, abordar a importância do controle do peso, da glicemia, pressão arterial, atenção ao nível dos colesteróis circulantes, dentre outros, de forma clara e adequada, segundo a realidade do indivíduo, para que este inicie a reflexão de suas ações cotidianas, colocando-se como protagonista do seu processo de saúde e doença.

O estudo apresentou como limitação o delineamento transversal que não possibilitou estabelecer relações de causa e o acompanhamento dos pacientes ao longo do tempo. Ademais, seus achados abrangem a identificação de fatores de risco para doenças cardiovasculares em pessoas que vivem com HIV em uso de TARV.

\section{AGRADECIMENTOS}

Agradecemos ao Fundo de Amparo à Pesquisa do Estado de São Paulo (FAPESP) pelo apoio na realização deste estudo. Número do processo: 2014/26843-7.

O presente trabalho foi realizado com apoio da Coordenação de Aperfeiçoamento de Pessoal de Nível Superior - Brasil (CAPES) - Código de financiamento 001

\section{REFERÊNCIAS}

1. Granjeiro A, Escuder MML, Castilho EA. Magnitude e tendência da epidemia de Aids em municípios brasileiros de 2002-2006. Rev. Saúde Pública. [Internet] 2010;44(3) [acesso em 04 set 2017]. Disponível: http://dx.doi.org/10.1590/ S0034-89102010005000013.

2. Miller TL. A próxima década: riscos cardiovasculares, desfechos, prevenção e tratamento da infecção pediátrica pelo HIV. J. Pediatr. [Internet] 2010;86(1) [acesso em 04 set 2017]. Disponível: http://dx.doi.org/10.1590/S002175572010000100002 .

3. Cioe PA, Crawford SL, Stein MD. Cardiovascular Risk-Factor Knowledge and Risk Perception Among HIV-Infected Adults. J. Assoc. Nurses AIDS Care. [Internet] 2014;25(1) [acesso em 04 set 2017]. Disponível: http://dx.doi. org/10.1016/j.jana.2013.07.006.

4. Ximenes RAA, Lacerda HR, Miranda-Filho DB, de Albuquerque MFPM, Montarroyos UR, Turchi MD, et al. Comparison between potential risk factors for cardiovascular disease in people living with HIV/AIDS in areas of Brazil. J. Infect. Dev. Ctries. [Internet] 2015;9(9) [acesso em 04 set 2017]. Disponível: http://dx.doi.org/10.3855/jidc.5867.

5. Joint United Nations Program on HIV/AIDS (UNAIDS). Global Aids Updates 2016. [Internet] Geneva: UNAIDS; 2016 [acesso em 04 set 2017]. Disponível: http://www.who.int/hiv/pub/arv/global-AIDS-update-2016_en.pdf?ua=1. 
6. Ministério da Saúde (BR). Secretaria de Vigilância em Saúde. Departamento de Vigilância, Prevenção e Controle das Infecções Sexualmente Transmissíveis, do HIV/Aids e das Hepatites Virais (DIAHV). Boletim Epidemiológico Aids DST. [Internet] Brasília: Ministério da Saúde; 2016 [acesso em 04 set 2017]. Disponível: http://www.aids.gov.br/pt-br/ taxonomy/term/595.

7. Zanni MV, Grinspoon SK. Hiv-specific immune Dysregulation and atherosclerosis. Curr HIV/AIDS Rep. [Internet] 2012;9(3) [acesso em 04 set 2017]. Disponível: http://dx.doi.org/10.1007/s11904-012-0123-y.

8. Grinspoon SK. Cardiovascular Disease in HIV: Traditional and Nontraditional Risk Factors. Top Antivir Med. [Internet] 2014;22(4) [acesso em 04 set 2017]. Disponível: https://www.ncbi.nlm.nih.gov/pubmed/25398068.

9. Nadel J, Holloway CJ. Screening and risk assessment for coronary artery disease in HIV infection: an unmet need. HIV Med. [Internet] 2017;18(4) [acesso em 04 set 2017]. Disponível: https://doi.org/10.1111/hiv.12422.

10. Ferrari TK, Cesar CLG, Alves MCGP, Barros MBA, Goldbaum M, Fisberg RM. Estilo de vida saudável em São Paulo, Brasil. Cad. Saúde Pública. [Internet] 2017;33(1) [acesso em 03 mar 2018]. Disponível: http://dx.doi.org/10.1590/ 0102-311x00188015.

11. World Health Organization (WHO). Diet, nutrition and the prevention of chronic diseases: report of a joint WHO/ FAO expert consultation. [Internet] Geneva: WHO; 2003 [acesso em 04 set 2017]. Disponível: http://apps.who.int/iris/ bitstream/10665/42665/1/WHO_TRS_916.pdf.

12. Kaplan RC, Kingsley LA, Sharret AR, Li X, Lazar J, Tien PC, et al. Ten-year predicted coronary heart disease risk in HIV-infected men and women. Clin Infect Dis. [Internet] 2007;45(8) [acesso em 04 set 2017]. Disponível: http://dx.doi. org/10.1086/521935.

13. Larré MC, Almeida ECS. Escore de Framingham na avaliação do risco cardiovascular em diabéticos. Rev. Rene. [Internet] 2014;15(6) [acesso em 04 set 2017] Disponível: http://dx.doi.org/10.15253/2175-6783.2014000600002.

14. Simão AF, Precoma DB, Andrade JP, Correa Filho H, Saraiva JFK, Oliveira GMM, et al. I Diretriz brasileira de prevenção cardiovascular da Sociedade Brasileira de Cardiologia - Resumo Executivo. Arq. Bras. Cardiol. [Internet] 2014;102(5) [acesso em 04 set 2017]. Disponível: http://dx.doi.org/10.5935/abc.2013S012.

15. Reis RK, Melo ES, Gir E. Fatores associados ao uso inconsistente do preservativo entre pessoas vivendo com HIV/aids. Rev. Bras. Enferm. [Internet] 2016;69(1) [acesso em 04 set 2017]. Disponível: http://dx.doi.org/10.1590/ 0034-7167.2016690106i.

16. de Moraes AS, Lopes DA, de Freitas ICM. Sex-specific differences in prevalence and in factors associated to the search for health services in a population based epidemiological study. Rev. bras. epidemiol. [Internet] 2014;17(2) [acesso em 04 set 2017]. Disponível: http://dx.doi.org/10.1590/1809-4503201400020004ENG.

17. Lima MAC, da Cunha GH, Galvão MTG, Rocha RP, Franco KB, Fontenele MSM. Systemic Arterial Hypertension in people living with HIV/AIDS: integrative review. Rev. Bras. Enferm. [Internet] 2017;70(6) [acesso em 07 mar 2018]. Disponível: http://dx.doi.org/10.1590/0034-7167-2016-0416.

18. Ding Y, Lin H, Liu X, Zhang Y, Wong FY, Sun YV, et al. Hypertension in HIV-Infected Adults Compared with Similar but Uninfected Adults in China: Body Mass Index-Dependent Effects of Nadir CD4 Count. AIDS Res Hum Retroviruses. [Internet] 2017;33(11) [acesso em 07 mar 2018]. Disponível: https://doi.org/10.1089/AID.2017.0008.

19. Tsai FJ, Cheng CF, Lai CH, Wu YC, Ho MW, Wang JH, et al. Effect of antiretroviral therapy use and adherence on the risk of hyperlipidemia among HIV-infected patients, in the highly active antiretroviral therapy era. Oncotarget. [Internet] 2017;8(63) [acesso em 07 mar 2018]. Disponível: https://doi.org/10.18632/oncotarget.22465.

20. Ministério da Saúde (BR). Secretaria de Atenção à Saúde. Departamento de Vigilância, Prevenção e Controle das Infecções Sexualmente Transmissíveis, do HIV/Aids e das Hepatites Virais (DIAHV). Protocolo clínico e diretrizes terapêuticas para manejo da infecção pelo HIV em adultos. [Internet] Brasília: Ministério da Saúde; 2013 [acesso em 07 mar 2018]. Disponível: http://www.aids.gov.br/pt-br/pub/2013/protocolo-clinico-e-diretrizes-terapeuticas-para-manejo-da-infeccao-pelo-hiv-em-adultos.

21. Melo ES. Risco cardiovascular e sua associação com variáveis demográficas, clínicas e psicossociais em pessoas vivendo com HIV/aids [dissertação]. Ribeirão Preto (RP): Universidade de São Paulo; 2016. 
22. Trevillyan JM, Hoy JF. Managing Cardiovascular Risk in People Living with HIV. Curr Treat Options Infect Dis. [Internet] 2016;8(2) [acesso em 04 set 2017]. Disponível: https://link.springer.com/article/10.1007/s40506-016-0071-y.

23. Reis RK, Melo ES, Galvão MTG, Gir E. Educação em saúde junto às pessoas com hiv/aids: proposta de intervenção multidisciplinar. Ciênc. cuid. saúde. [Internet] 2014;13(3) [acesso em 04 set 2017]. Disponível: https://dx.doi. org/10.4025/cienccuidsaude.v13i3.21120. 